\title{
EXTRAÇÃO DE MICRONUTRIENTES EM SOLO COM SOLUÇÃO DE DTPA EM FORNO DE MICROONDAS COM RADIAÇÃO FOCALIZADA ${ }^{(1)}$
}

\author{
M. C. C. LOMBARDI ${ }^{(2)}$, J . A. NÓBREGA ${ }^{(3)}$ \& A. R. A. NOGUEIRA ${ }^{(4)}$
}

\begin{abstract}
RESUMO
Neste trabal ho, é proposto um procedi mento alternativo para a extração de micronutrientes em amostras de solo para fins de avaliação da fertilidade. Amostras provenientes de programa colaborativo de análise de solos, com diferentes características físicas e químicas, foram submetidas à extração com ácido dietilenotriaminopentaacético (DTPA), empregando-se energia de microondas com radiação focalizada. Os resultados indicaram uma redução significativa no tempo requerido para extração de $\mathrm{Cu}, \mathrm{Mn}$ e $\mathrm{Zn}$. Os resultados apresentaram uma redução si gnificativa no tempo de preparo das amostras: de $\mathbf{1 2 0} \mathbf{m i n}$, no procedimento convencional envolvendo agitação à temperatura ambiente, para $10 \mathrm{~min}$, no procedimento assistido por microondas. Não foi possível estabelecer um procedimento único para a extração si multânea também de $\mathrm{Fe}$, em virtude de sua maior liberação com essa forma de aquecimento. Entretanto, demonstrou-se que o aquecimento no forno de microondas tem potencial para utilização na extração de micronutrientes em análise de solo de rotina.
\end{abstract}

Termos de indexação: extração assistida por microondas, frascos abertos, avaliação de fertilidade, solo.

\footnotetext{
(1) Parte da Tese de Doutorado do primeiro autor, apresentada ao Programa de Pós-Graduação em Química da Universidade Federal de São Carlos - UFSCar. Trabal ho realizado com apoi o financeiro da FAPESP (processos 97/04324-0, 98/10814-3). Recebido para publicação em fevereiro de 2001 e aprovado em março de 2002.

(2) Doutor em Química, Departamento de Química, Universidade Federal de São Carlos - UFSCar. Caixa Postal 676, CEP 13560970 São Carlos (SP). E-mail: amadeuln@terra.com.br

(3) Professor Departamento de Química, UFSCar. E-mail: djan@erra.com.br

(4) Pesquisadora da Embrapa Pecuária Sudeste. Caixa Postal 339, CEP 13560-970 São Carlos (SP). E-mail:anarita@cppse.embrapa.br
} 


\title{
SUMMARY: FOCUSED MICROWAVE-ASSISTED PROCEDURE FOR MICRONUTRIENT EXTRACTION FROM SOIL SAMPLES WITH DTPA SOLUTION
}

\begin{abstract}
This study presents an alternative procedure for the extraction of micronutrients from soil samples for fertility evaluation. Samples from an interdisciplinary program for soil analysis with different physical and chemical characteristics were subjected to DTPA extraction using focused microwave radiation energy. The microwaveassisted procedure (10 min) proved to beremarkably timesaving for $\mathrm{Cu}, \mathrm{Mn}$, and $\mathrm{Zn}$ extraction when compared to thetimeconsume of conventionally prepared samples (120 min), using mechanical sti rring at room temperature. The devel opment of a single procedurefor si multaneous extraction of Fe was not possible, however, due to excessive Fe release during the heating process. Nevertheless, the feasibility of microwave-assisted procedures in routine analyses was validated.
\end{abstract}

Index terms: mi crowave-assisted extraction, open flasks, fertility eval uation, soil.

\section{INTRODUÇÃO}

Na literatura, são descritos diferentes procedimentos para a determinação de metais em solos, com vistas em medir a concentração total dos el ementos (K owal ewka et al., 1998; M cGrath, 1998) ou a forma na qual eles estão presentes na amostra.

A variedade de procedimentos existentes para a decomposição da amostra (K owal ewka et al., 1998) e a falta de uniformidade nos procedimentos empregados para extração dos elementos dificultam a interpretação e a comparação dos resultados obtidos por diferentes laboratórios. De acordo com Quevauviller (1998), os resultados de extração somente são úteis e aplicáveis ao estudo ambiental, se forem usados procedimentos de extração bem definidos eaceitos. Uma visãogeral dos procedimentos deextraçãosimples eseqüencial mais freqüentemente usados foi apresentada por Rauret (1998).

Diversos fatores influem na eficiência de extração, principalmente aqueles inerentes ao sistema solo-planta. O interesse de técnicos e agricultores pela análise dos principais micronutrientes ( $B, C u, F e, M n$ e $Z n$ ) em solos tem aumentado a cada ano. A maior procura por esse serviço deve-se ao aparecimento crescente de diversas deficiências nutricionais, que vêm sendo observadas principalmente pela incorporação de sol os de baixa fertilidade ao sistema produtivo e ao uso de variedades mais produtivas e, em decorrência disso, com maiores exigências minerais (Abreu et al., 1997; Raij et al., 2001).

Na literatura, diversas soluções são empregadas para simular a absorção dos micronutrientes pelas plantas, como Mehlich-1 e 3, ácido clorídrico, ácido dietilenotriaminopentaacético (DTPA), ácido etilenodiaminotetraacético (EDTA), doreto de cálcio e acetato de amônio, dentre outras. Todas essas soluções foram propostas com o objetivo de estabel ecer qual delas possibilita mel hor simulação da ação das raízes (Silva, 1999).

O método oficial empregado no estado de São Paulo para a extração de $\mathrm{Cu}, \mathrm{Fe}, \mathrm{Mn}$ e $\mathrm{Zn}$ é o de Lindsay \& Norvel (1978), que usa solução de DTPA como extrator e vem sendo adotado desde 1994, quando foi escol hido durantea reunião do Programa de Controle de Qualidade de Análise de Solo, coordenado pelo I nstituto Agronômico de Campinas, IAC (Raij et al., 2001). Esse extrator vem apresentando as melhores correlações entre os micronutrientes presentes no solo e os teores nas plantas, sendo um agente quelante adequado para complexar simultaneamente $\mathrm{Cu}, \mathrm{Fe}, \mathrm{Mn}$ e $\mathrm{Zn}$. Combina-se com os íons livres desses elementos, formando complexos solúveis, e reduz suas atividades em solução. Como conseqüência, os íons dessorvem da superfície dos colói des ou solubilizamse a partir da fase sólida para reabastecer os íons em solução. Os teores de $\mathrm{Cu}, \mathrm{Fe}, \mathrm{Mn}$ e $\mathrm{Zn}$ complexados acumulados na solução durante a extração dependem da atividade desses íons livres na solução do sol o (fator intensidade), da habilidade do sol o em reabastecer a sol ução (fator capacidade), da estabili-dade do quelato e da capacidade do complexante em competir com a matéria orgânica pel o íon (Lindsay \& N orwell, 1978).

Procedimentos com microondas desenvolvidos têm sido descritos na literatura, empregando-se extração de íons metálicos em amostras de solo e sedimentos usando água régia $\left(\mathrm{HCl}: \mathrm{HNO}_{3}-3: 1 \mathrm{v} / \mathrm{V}\right)$. Nieuwenhuize \& Poley-vos (1991) relataram vantagens deste método em relação ao procedi mento convencional de extração, como a rapidez e a diminuição dos riscos de contaminação decorrentes do emprego de frascos fechados. 
Lorentzen \& Kingston (1996) compararam a eficiência de lixiviação de $\mathrm{Cd}, \mathrm{Cr}, \mathrm{Cu}, \mathrm{Ni}, \mathrm{Pb}$ e $\mathrm{Zn}$ usando o método EPA 3050B, que é o procedimentopadrão de preparo de amostras em chapa de aquecimento indicado pela Agência de Proteção Ambiental Americana (Edgell, 1988), e adaptaram esse procedimento para efetuar a extração por microondas. Nesse trabalho, diferentes fornos de microondas com controle de temperatura e pressão disponíveis comercialmente foram testados. Os elementos foram lixiviados com $\mathrm{HNO}_{3}, \mathrm{H}_{2} \mathrm{O}_{2}$ e $\mathrm{HCl}$ e determinados por espectrometria de absorção atômica com chama ou forno de grafite. Os autores concluíram que o emprego controlado de energia de microondas é uma alternativa eficiente em relação às fontes de aquecimento convencionais recomendadas pelo método EPA 3050B. Além de apresentar resultados mais reprodutíveis, o tempo gasto no preparo foi significativamente reduzido.

Apesar desses resultados favoráveis, que demonstram a eficiência desses extratores na recuperação de vários el ementos nos solos, ainda é pequeno o número de trabalhos publicados com digestão ou extração assistida por microondas, usando soluções ácidas diluídas ou bases orgânicas complexantes. A qualidade do resultado de uma determinação analítica pode ser comprometida por erros sistemáticos introduzidos durante o processo de pré-tratamento da amostra, como a contaminação pelo material do frasco durante a manipulação e armazenagem. Outro aspecto que deve ser considerado é a falta de controle individual de temperatura nos frascos quando é feito o aquecimento condutivo.

Assim, no presenteestudo, avaliou-se procedimento de extração de micronutrientes em amostras desolo empregando DTPA e aquecimento assistido por microondas com radiação focalizada, que opera à pressão ambiente e permite a utilização de maior volume de amostras e reagentes sem os riscos de explosão observados quando se trabalha com sistemas fechados (Kingston \& Haswell, 1997).

\section{MATERIAL E MÉTODOS}

\section{Instrumentação e condições de operação}

As extrações com DTPA foram realizadas em forno de mi croondas com radiação focalizada model o Star System $6^{\mathrm{TM}}$ (CEM) com potência nominal de $950 \pm 50 \mathrm{~W}$. Esse equipamento possui um único magnetron, propagando as microondas ali geradas por meio de um guia de ondas até à cavidade. A quantidade de energia das microondas incidentes é controlada pela abertura de fendas posicionadas em cada cavidade (Figura 1). A potência e o tempo de abertura de cada fenda são estabelecidos pela temperatura selecionada na programação do equipamento. Sensores deinfravermel ho posicionados abaixo de cada frasco reacional possibilitam o controle de temperatura.

As determinações foram realizadas com um espectrofotômetro de absorção atômica com chama Gemini, I ntralab, modelo AA12/1475, empregando chama ar-acetileno e lâmpadas de catodo oco como fonte de radiação. Os comprimentos de onda utilizados foram de 324,7; 248,3; 279,5 e 213,9 nm, respectivamente, para $\mathrm{Cu}, \mathrm{Fe}, \mathrm{Mn}$ e Zn.

\section{Reagentes, soluções e amostras}

Os reagentes empregados foram degrau analítico (PA). As ponteiras e os frascos usados para o preparo e estocagem de soluções de referência e amostras foram descontaminados em banhos que continham solução de $\mathrm{HNO}_{3} 10 \%(\mathrm{v} / \mathrm{v})$, durante $24 \mathrm{~h}$.

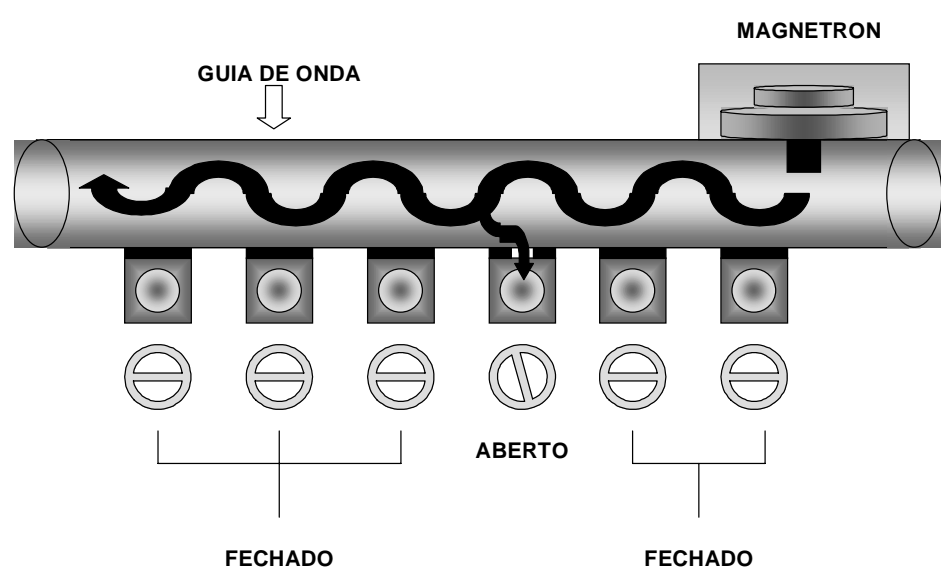

Figura 1. Esquema do guia de ondas do forno de microondas com radiação focalizada equi pado com um único magnetron. 
A solução empregada para extração dos micronutrientes do sol o foi preparada pela mistura das soluções $5 \times 10^{-3} \mathrm{~mol} \mathrm{~L}^{-1}$ de ácido dietilenotriaminopentaacético (DTPA: [ $\left.(\mathrm{HOCOCH})_{2} \mathrm{NCH}_{2}\right]_{2}$ $\mathrm{NCH}_{2} \mathrm{COOH}$ ); 0,1 mol L-1 de trietanolamina (TEA) e $1,0 \times 10^{-2} \mathrm{~mol} \mathrm{~L}^{-1}$ de cloreto de cál cio $\left(\mathrm{CaCl}_{2} \cdot \mathrm{H}_{2} \mathrm{O}\right)$ e o pH foi ajustado para 7,3 com solução 4,0 mol L-1 $\mathrm{HCl}$ (Lindsay \& Norwell, 1978).

A curva analítica mista foi preparada em meio de DTPA a partir de diluições apropriadas de soluções estoque que continham $1.000 \mu \mathrm{g} \mathrm{L}-1$, preparadas a partir de $\mathrm{Mn}$ metálico e $\mathrm{Cu}$, Fe e Zn a partir da diluição de ampolas concentradas comerciais.

Foram utilizadas amostras de solo do Programa de Controle de Análise de Solo, coordenado pelo Instituto Agronômico (IAC). Essas amostras, com diferentes características, foram previamente analisadas nos diferentes laboratórios participantes do programa, que utilizam o procedimento convencional para extração de $\mathrm{Cu}, \mathrm{Fe}, \mathrm{Mn}$ e $\mathrm{Zn}$, descrito por Lindsay \& Norwell (1978): $10 \mathrm{~g}$ de amostra receberam $30 \mathrm{~mL}$ de solução DTPA, sendo, a seguir, agitadas durante 120 min. Após esse período, as amostras foram filtradas com papel de filtro Whatman n०. 42. O tempo gasto na filtração por gravidade depende da granulometria do solo, podendo ser consi derados 60 min como tempo médio gasto nesta etapa do procedimento. Os teores médios obtidos entre os diferentes laboratórios, assim como os valores, máximo e mínimo, aceitáveis para o el emento químico em cada amostra, fornecidos pelo Programa Interlaboratorial, foram considerados como valores de referência e comparados aos obtidos com o método proposto neste trabal ho (Quadro 1).

\section{Procedimento Experimental}

Simulando o procedimento empregado no estudo interlaboratorial, massas de 3,3 g deamostra de sol o foram transferidas para os frascos de vidro borossilicato usados no forno de microondas. A seguir, adicionaram-se $10 \mathrm{~mL}$ de solução extratora deDTPA.

Para avaliar o melhor procedimento a ser empregado para a extração por microondas, foram realizados ensaios variando-se o tempo e a temperatura (rampa e patamar) do programa de aquecimento, com o objetivo de obter a extração simultânea de todos os elementos dentro da faixa estatisticamenteaceitável. Os mel hores resultados

Quadro 1. Teores de Cu, Fe, Mn e Zn extraídos em cinco amostras de solo com solução de DTPA. Valores médios e desvios-padrão $(n=3)$ determi nados pelo procedimento proposto, assistido por microondas e pelo método convencional

\begin{tabular}{|c|c|c|c|c|c|}
\hline Amostra & Procedimento & $\mathrm{Cu}$ & $\mathbf{F e}$ & Mn & Zn \\
\hline & & & & -1 & \\
\hline \multirow[t]{2}{*}{1} & $\begin{array}{l}\operatorname{Vm}(1) \\
\operatorname{Vr}(2)\end{array}$ & $\begin{array}{l}1,79 \pm 0,08 \\
1,61 \pm 0,27\end{array}$ & $\begin{array}{c}129,7 \pm 6,5 \\
73,6 \pm 13,9\end{array}$ & $\begin{array}{l}1,77 \pm 0,05 \\
1,85 \pm 0,40\end{array}$ & $\begin{array}{l}0,86 \pm 0,04 \\
0,88 \pm 0,18\end{array}$ \\
\hline & $F^{(3)}$ & $(1,0-2,2)$ & $(46-101)$ & $(1,2-2,5)$ & $(0,6-1,2)$ \\
\hline \multirow[t]{2}{*}{2} & $\begin{array}{l}\operatorname{Vm} m^{(1)} \\
V r^{(2)}\end{array}$ & $\begin{array}{l}2,33 \pm 0,14 \\
2,29 \pm 0,36\end{array}$ & $\begin{array}{r}111,2 \pm 2,8 \\
51,7 \pm 21,6\end{array}$ & $\begin{array}{l}3,41 \pm 0,21 \\
2,90 \pm 0,51\end{array}$ & $\begin{array}{l}1,01 \pm 0,05 \\
1,06 \pm 0,20\end{array}$ \\
\hline & $F^{(3)}$ & $(1,6-3,0)$ & $(30-73)$ & $(1,9-3,9)$ & $(0,7-1,5)$ \\
\hline \multirow[t]{2}{*}{3} & $\begin{array}{l}\operatorname{Vm}(1) \\
V r^{(2)}\end{array}$ & $\begin{array}{l}5,13 \pm 2,30 \\
4,15 \pm 0,54\end{array}$ & $\begin{array}{l}53,7 \pm 5,2 \\
17,6 \pm 7,6\end{array}$ & $\begin{array}{l}57,6 \pm 2,8 \\
49,54 \pm 18,64\end{array}$ & $\begin{array}{l}1,55 \pm 0,14 \\
1,52 \pm 0,26\end{array}$ \\
\hline & $F^{(3)}$ & $(3,1-5,2)$ & $(10-25)$ & $(33,8-65,3)$ & $(1,0-2,0)$ \\
\hline \multirow[t]{2}{*}{4} & $\begin{array}{l}\operatorname{Vm}(1) \\
\operatorname{Vr}(2)\end{array}$ & $\begin{array}{l}1,11 \pm 0,07 \\
1,09 \pm 0,17\end{array}$ & $\begin{array}{r}122,6 \pm 1,8 \\
74,8 \pm 16,2\end{array}$ & $\begin{array}{l}11,1 \pm 0,07 \\
11,02 \pm 1,78\end{array}$ & $\begin{array}{l}0,73 \pm 0,02 \\
0,73 \pm 0,16\end{array}$ \\
\hline & $\mathrm{FA}^{(3)}$ & $(0,8-1,4)$ & $(51-99)$ & $(7,5-14,6)$ & $(0,5-1,0)$ \\
\hline \multirow[t]{2}{*}{5} & $\begin{array}{l}\operatorname{Vm}(1) \\
\operatorname{Vr}(2)\end{array}$ & $\begin{array}{l}4,10 \pm 0,20 \\
5,07 \pm 0,54\end{array}$ & $\begin{array}{l}82 \pm 7,0 \\
19,3 \pm 3,6\end{array}$ & $\begin{array}{l}54,2 \pm 0,2 \\
75,8 \pm 12,6\end{array}$ & $\begin{array}{l}3,4 \pm 0,1 \\
4,88 \pm 0,81\end{array}$ \\
\hline & $F^{(3)}$ & $(4,0-6,1)$ & $(12-27)$ & $(50,7-100,9)$ & $(3,3-6,5)$ \\
\hline
\end{tabular}

Consideradas as faixas aceitáveis para cada amostra, resultados estatisticamente concordantes a 95 \% pelo Teste t. Exceção para as amostras de Fe.

${ }^{(1)}$ Extração assistida por microondas. ${ }^{(2)}$ Valor de referência. ${ }^{(3)}$ Faixa aceitável. Referem-se às médias e aos valores, máximo e mínimo, estatisticamente aceitáveis dos resultados obtidos a partir das determinações realizadas pel os laboratórios que utilizam o DTPA como extrator e participam do Programa de Controle de Análise de solo coordenado pelo I nstituto Agronômico (IAC). 
foram obtidos com o tempo de dois minutos para atingir a temperatura de $98{ }^{\circ} \mathrm{C}$ (rampa), sendo essa temperatura mantida durante oito min (patamar). O tempo de dois minutos para atingir a temperatura necessária permitiu que o aquecimento fosse realizado lentamente, evitando a ebulição violenta mediante um aquecimento brusco, que provocaria o espal hamento da amostra nas paredes internas do frasco, prejudicando o processo de extração.

Após o término do programa de aquecimento, aguardou-se o resfriamento até atingir a temperatura de aproximadamente $80 \stackrel{\circ}{\circ}$. Em seguida, a mistura foi centrifugada a $4.000 \mathrm{rpm}$, durante $10 \mathrm{~min}$, e os teores de $\mathrm{Cu}, \mathrm{Fe}, \mathrm{Mn}$ e $\mathrm{Zn}$ presentes no extrato foram determinados por espectrometria de absor ção atômica com chama.

\section{RESULTADOS E DISCUSSÃO}

Os resultados obtidos para $\mathrm{Cu}, \mathrm{Mn}$ e $\mathrm{Zn}$ foram concordantes com as médias dos resultados do programa intelaboratorial a $95 \%$ de confiança para todas as amostras usando teste-t pareado, exceto para Cu e Mn na amostra 5 (Quadro 1). Todavia, quando considerados os val ores (máximo e mínimo) aceitáveis para as amostras, esses valores também apresentaram nível de confiança $\geq 95 \%$ (Quadro 1). Os teores de Fe encontrados para todas as amostras estudadas foram superiores aos valores de referência, o que inviabiliza a utilização do procedimento assistido por microondas para a extração simultânea de Cu, Mn, Zn eFe. Diminuindo o tempo ou a temperatura de extração para otimizar as condições para o $\mathrm{Fe}$, ocorreu uma baixa recuperação de $\mathrm{Mn}$. Aumentando o tempo ou a temperatura de extração, concentrações ainda mais elevadas de Fe foram obtidas.

De acordo com Page et al. (1982), o procedimento com DTPA éuma extração que não está em equilíbrio e, portanto, fatores, tais como: tempo, vel ocidade de agitação e geometria dos frascos reacionais são críticos para a determinação quantitativa de Fe. Portanto, para que os resultados possam ser comparados pel os diferentes laboratóri os de anál ise, esses fatores devem ser padronizados.

Com base nesses resultados, é possível antever a potencial idade do emprego de aquecimento assistido por microondas, com vistas em extrair os el ementos importantes para a correta avaliação da fertilidade do solo. Os casos de deficiência de Fe em São Paulo são bastante raros, sendo poucos os estudos que procuram avaliar a correlação entre os teores desse micronutriente no solo e sua ação como fator de produção na planta. Normalmente, são utilizadas soluções extratoras como DTPA ou Mehlich para a determinação de Fe (Raij et al., 2001).
Com o procedimento proposto, evita-se filtração, o que resulta em redução das perdas e da contaminação. Além disso, el imina-se o tempo gasto na filtração do extrato empregando-se filtração por gravidade, que pode ser superior a $60 \mathrm{~min}$, dependendo do teor de argila da amostra.

\section{CONCLUSÕES}

1. O procedimento proposto apresenta-se como uma al ternativa ao método convencional de extração por agitação à temperatura ambiente para a determinação de $\mathrm{Cu}, \mathrm{Mn}$ e $\mathrm{Zn}$ em amostras de solo com solução de DTPA.

2. A principal vantageméa significativa redução no tempo de extração, que é de $120 \mathrm{~min}$, no procedimento convencional, e de $10 \mathrm{~min}$, no procedimento assistido por microondas. Quando se considera também a substituição da filtragem pela centrifugação, um ganho de até 160 min pode ser obtido, além da diminuição da possibilidade de contaminação.

\section{AGRADECIMENTOS}

Os autores agradecem o apoio financeiro da FAPESP (Processo 98/10814-3). M.C.C. Lombardi; à FAPESP, pela concessão da bol sa de doutoramento (Processo 97/04324-0). A.R.A. Nogueira e J.A. Nóbrega são gratos ao CNPq, pelas bolsas de produtividade concedidas.

\section{LITERATURA CITADA}

ABREU, C.A.; ABREU, M.F., HARADA, L.S. \& ANDRADE, J.C. The effects of the DTPA extraction conditions on the determination of micronutrients in Brazilian soils. Comm. Soil Sci. Plant Anal., 28:1-11, 1997.

EDGELL, K. \& USEPA - UNITED STATES ENVIRONMENTAL PROTECTION AGENCY - Method Study 37 - SW-846 Method 3050 Acid Digestion of Sediments, Sludges, and Soils. EPA Contract No. 68-03-3254, Washington, DC, Gov. Print Office, 1988.

KINGSTON, H.M. \& HASWELL, S.J ., eds. Microwave-enhanced chemistry - Fundamentals, sample preparation and applications, Washington, American Chemical Society, 1997. $772 p$.

KOWALEWSKA, Z.; BULSKA, E. \& HULANICKI,A. The effect of sample preparation on metal determination in soil by FAAS. Talanta, 46:439-448, 1998.

LINDSAY, W.L. \& NORWELL, W. Development of a DTPA soil test for zinc, iron, manganese and copper. Sci. Soc. Am. J ., 42:421- 428, 1978. 
LORENTZEN, E.M.L. \& KINGSTON, H.M., Comparison of microwave-assisted and conventional leaching using EPA methods 3050B. Anal. Chem., 68:4316-4320, 1996.

McGRATH,D. Use of microwave digestion for estimation of heavy metal content of soil in a geochemical survey. Talanta, 46:439-448, 1998.

NIEUWENHUIZE, J.\& POLEY-VOS, C. Comparison of microwave and conventional extraction techniques for the determination of metals in soil, sedment and sludgesamples by atomic spectrometry. Analyst, 116:347-351, 1991.

PAGE, A.L.; MILLER, R.H. \& KEENEY, D.R. Methods of soil analysis. Part 2. Chemical and Microbiological Properties. Madison, American Society of Agronomy, 1982. 1159p.
QUEVAUVILLER, P. Operationally defined extracion procedures for soil and sediment analysis - II. Certified reference materials. Trends Anal. Chem., 17:632-642, 1998.

RAIJ , B. VAN;ANDRADE,J .C.; CANTARELLA, H. \& QUAGGIO, J.A., eds., Análise química para avaliação da fertilidade de solos tropicais. Campinas, I nstituto Agronômico, 2001. 285p.

RAURET, G. Extraction procedures for the determination of heavy metals in contaminated soil and sediment. Talanta, 46:449-455, 1998.

SILVA, F.C. org. Manual de análises químicas de solos, plantas e fertilizantes. Brasília, EMBRAPA Solos, EMBRAPA Informática Agropecuária, 1999. 370p. 